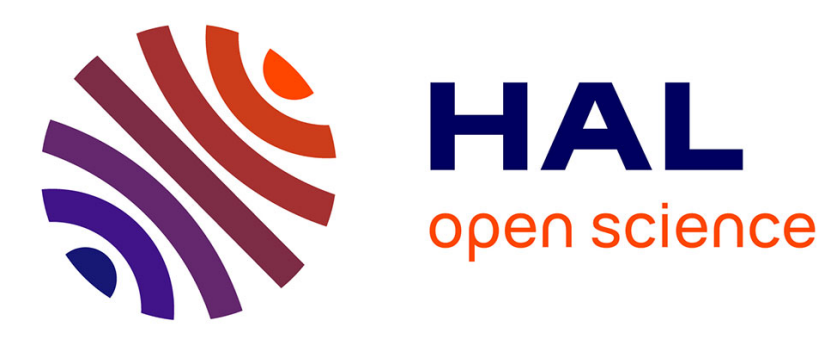

\title{
Mobility of Domain Walls in Proper Ferroelastic Martensites
}

\author{
G. Barsch
}

\section{To cite this version:}

G. Barsch. Mobility of Domain Walls in Proper Ferroelastic Martensites. Journal de Physique IV Proceedings, 1995, 05 (C8), pp.C8-119-C8-124. 10.1051/jp4:1995813 . jpa-00254062

\section{HAL Id: jpa-00254062 https://hal.science/jpa-00254062}

Submitted on 1 Jan 1995

HAL is a multi-disciplinary open access archive for the deposit and dissemination of scientific research documents, whether they are published or not. The documents may come from teaching and research institutions in France or abroad, or from public or private research centers.
L'archive ouverte pluridisciplinaire HAL, est destinée au dépôt et à la diffusion de documents scientifiques de niveau recherche, publiés ou non, émanant des établissements d'enseignement et de recherche français ou étrangers, des laboratoires publics ou privés. 


\title{
Mobility of Domain Walls in Proper Ferroelastic Martensites
}

\author{
G.R. Barsch
}

The Pennsylvania State University, Intercollege Materials Research Laboratory and Department of Physics, University Park, PA 16801, U.S.A.

\begin{abstract}
Based on the Landau-Ginzberg free energy functional for an $\mathrm{O}_{h}-\mathrm{D}_{4 h}$ proper ferroelastic martensitic transformation the mobility of a (110) twin boundary in a large bicrystal has been calculated by including dissipation in the approximation of the phonon viscosity model and by solving the inverse boundary value problem for the limiting case of strong shear modulus softening. Application to actual materials requires determination of the phonon viscosity tensor from experimental ultrasonic attenuation or low frequency internal friction data after subtraction of "extrinsic" losses, especially those from pretransformation structural strain modulations ("tweed") and dislocations. Numerical application to $\mathrm{V}_{3} \mathrm{Si}$, the only proper ferroelastic martensite for which such experimental data pertaining to the soft [110]/110] shear mode are available, is discussed.
\end{abstract}

\section{INTRODUCTION}

The mobility of martensitic interfaces is a crucial link in the causal chain that determines the kinetics of martensitic transformations [1,2]. However, in spite of their importance for the nucleation and growth of the martensite product phase, so far single interface mobility measurements have been carried out only for $\mathrm{Cu}-\mathrm{Al}-\mathrm{Ni}$ alloy single crystals [3,4]. By using a dislocation model for martensitic interfaces [5] and utilizing available models for the mobility of dislocations [6] Grujicic, Olson and Owen [1,7] have described their experimental results [4] successfully in terms of the interface interaction with heterophase inclusions and premartensitic features (tweed).

Other theoretical studies of moving martensitic interfaces have been based on nonlinear elastic continuum models in one dimension (1D) [8-11]. In these models martensitic interfaces are described either as topological solitons* or as discontinuities in the strain gradients (i.e. shock waves), but dislocations are included, if at all, only implicitly through the postulated form of the dependence of the interface mobility on the driving force.

The present work was motivated by the fact that, on the one hand, planar, stress free and coherent martensitic interfaces in three dimensions (3D) can be described consistently in terms of topological solitons without interface dislocations [12-14], and that, on the other hand, the strain fields and the dynamic behaviour of martensitic interfaces and of dislocations are quite different. Specifically, for dislocations the strain field decreases as an inverse power of distance, and the effective mass (per unit length) is for small velocities (< the shear velocity for screw dislocations, and < the Rayleigh velocity for edge dislocations) given by $[15] \rho^{2} / 2$ ( $\rho=$ crystal density; $b=$ Burgers vector), whereas for twin boundaries (TB's) the strain field decreases exponentially with distance $[12] \times[\sim \exp (-\mathrm{x} / \xi) ; 2 \xi=\mathrm{TB}$ width], and the effective mass (per unit area) is given by [16] $\rho(D-2 \xi)$ where D is the separation of TB's in a twin band. Therefore, in the present work the stress induced motion of twin boundaries in the tetragonal martensite phase is studied by extending a previous Landau-Ginzburg (LG) model for the $O_{h}-D_{4 h}$ proper ferroelastic (FE) transformation [12-14] by including dissipation in the classical phenomenological form [17,18]. This particular form has been justified microscopically in terms of the intrinsic attenuation an acoustic wave experiences as a result of its interaction with the thermal phonons $[15,18]$ and is therefore referred as the "phonon viscosity" model.

*The term "soliton" is used here in a generic sense so as to include solitary waves. 
Future extension of the present model for the TB mobility to parent/product interfaces and inclusion of interface-defect interactions will allow comparison with the results based on the interface dislocation model $[2,7]$.

\section{THEORETICAL MODEL}

\subsection{Landau-Ginzburg Free Energy and Static Solutions}

The Landau free energy (LFE) for a proper ferroelastic martensitic transformation from a cubic $\left(\mathrm{O}_{h}\right)$ to a tetragonal ( $D_{4 h}$ ) phase is (for $e_{1}=0$, i.e. small volume change) given by $[12,13]$ :

$$
\Phi_{L}=A\left(e_{2}^{2}+e_{3}^{2}\right)+B e_{3}\left(e_{3}^{2}-3 e_{2}^{2}\right)+C\left(e_{2}^{2}+e_{3}^{2}\right)^{2}
$$

The two-component order parameter $\left(e_{2}, e_{3}\right)$ consists of the deviatoric strains that are part of the full set of strain symmetry coordinates for cubic symmetry, viz.

$$
\begin{aligned}
& e_{1}=\left(\eta_{11}+\eta_{22}+\eta_{33}\right) / \sqrt{3} ; e_{2}=\left(\eta_{11}-\eta_{22}\right) / \sqrt{2} ; e_{3}=\left(\eta_{11}+\eta_{22}-2 \eta_{33}\right) / \sqrt{6} \\
& e_{4}=\eta_{23}=\eta_{32} ; e_{5}=\eta_{13}=\eta_{31} ; e_{6}=\eta_{12}=\eta_{21}
\end{aligned}
$$

Here $\eta_{i j}=(1 / 2)\left(u_{i, j}+u_{j, i}+u_{k, i} u_{k, j}\right)$ are the components of the Lagrangian strain tensor, and $u_{i, j}=\left(\partial u_{i} / \partial x_{j}\right)$ the derivatives of the displacement components $u_{i}$ w.r.t. the material coordinates $x_{j}$ along the directions of the cubic crystallographic axes $(i, j, k=1,2,3$; summation convention). Assuming the usual linear temperature variation of the "soft" shear modulus A and introducing the dimensionless temperature $\tau$ leads to

$$
A=\left(c_{11}-c_{12}\right) / 2=A_{0} \tau ; A_{0}=B^{2} / 4 C ; \tau=\left(T-T_{c}\right) /\left(T_{0}-T_{c}\right)
$$

where $T_{0}(\tau=1)$ and $T_{c}(\tau=0)$ denote the transformation temperature and the mechanical stability limit of the cubic parent phase, respectively. Note that $C>0$ always. As in Refs. [13,14] we consider or assume (i) geometric linearity (i.e. neglecting the third term in $\eta_{i j}$ ); (ii) a dislocation free crystal (i.e. validity of the strain compatibility conditions); (iii) the mixed inverse-direct boundary value problem implied in the assumption that the strain field of a martensitic interface depends only on the coordinate perpendicular to the interface; (iv) sufficiently "soft" shear modulus at $T_{0}$, specified by $A_{0} \ll 3\left(2 K+c_{44}\right)$ where $K$ is the bulk modulus and $c_{44}$ the second ("hard") shear modulus of the cubic phase. For the particular temperature $\tau=-9$ this assumption is not necessary [12]. It has been shown [13,14] that under these assumptions the contributions from the other symmetry coordinates do not enter and that the strain energy is entirely determined by the LFE, Eq. (1), and it will be shown below that in this case exact quasi-1D solutions of the equations of motion in $3 D$ for the displacements $u_{i}=u_{i}(\bar{x}, t)$ exist that describe a TB moving under the influence of external forces.

In Figs. 1a and $2 a$ the contours of $\Phi_{\mathrm{L}}\left(\mathrm{e}_{2}, \mathrm{e}_{3}\right)=$ const are shown for two different temperatures. There are three minima, labeled I, II and III, that correspond to the three tetragonal variants of the martensite phase with the c-axis along the $x_{2}, x_{1}$ and $x_{3}$ axes, respectively. Their (temperature dependent) positions are:

$$
\left(e_{2}, e_{3}\right)=( \pm \sqrt{3}, 1) e^{*} ;(0,-1)\left(2 e^{*}\right),
$$

correspondingly, where

$$
\mathrm{e}^{*}=3 \mathrm{Bz} / 16 \mathrm{C}=(3 / 8) \sqrt{\mathrm{A}_{0} / \mathrm{C}} \mathrm{z}=(\mathrm{c} / \mathrm{a}-1) / \sqrt{6} ; \mathrm{z}=[1+\sqrt{1-8 \tau / 9}]
$$

$a$ and $c$ denote the lattice parameters of the tetragonal phase; $e_{2}=e_{3}=0$ correspond to the cubic parent phase.

In order to describe a planar (1T0)TB between two tetragonal variants we consider a " $V$ "-shaped bicrystal that results from the $\mathrm{O}_{\mathrm{h}}-\mathrm{D}_{4 \mathrm{~h}}$ transformation of a rectangular parallelepiped with edges parallel to the axes of a rectangular coordinate system $\left(r, s, x_{3}\right)$, where $r, s, x_{3}$ are along [110], [1T0] and [001] of the cubic parent phase, respectively. Then the s-axis is normal to the (110) TB plane. In general, i.e. without assumption (iv), the TB is described by the order parameter profile $\left\{e_{2}(s), e_{3}(s)\right\}$ that connects the two minima I and II. However, for $\tau=-9$, or when the above conditions (i) to (iv) are satisfied the two minima are connected by a straight line, i.e. $e_{3}=e_{3}^{*}=$ const, and the strain profile is obtained from the LG free energy for $e=e_{2}$ given by $[13,14]$

$$
\Phi_{L G}\left(e, e^{\prime}\right)=\Phi_{L}^{*}(e)+g(d e / d s)^{2}
$$


Figure 1 and 2. (a) Contours of constant Landau free energy $\Phi_{L}\left(e_{2}, e_{3}\right)$ as function of the deviatoric strains for $\tau=-9$ and $\tau=1$, respectively; $\left(e_{2}, e_{3}\right)$ are in units of $B / 2 C$. (b) Effective Landau free energy $\Phi_{L}\left(e_{2}, e_{3}^{*}\right)$ (in units of $B^{4} / 64^{3}$ ) for $e_{3}^{*}=$ const, representing an elastic " $\phi^{4 "}$. model for a twin boundary between tetragonal variants I and II for $\tau=-9$ and, for sufficiently small soft shear modulus, for arbitrary temperature $\tau \leq 1$ (Refs. [12-14]).

where

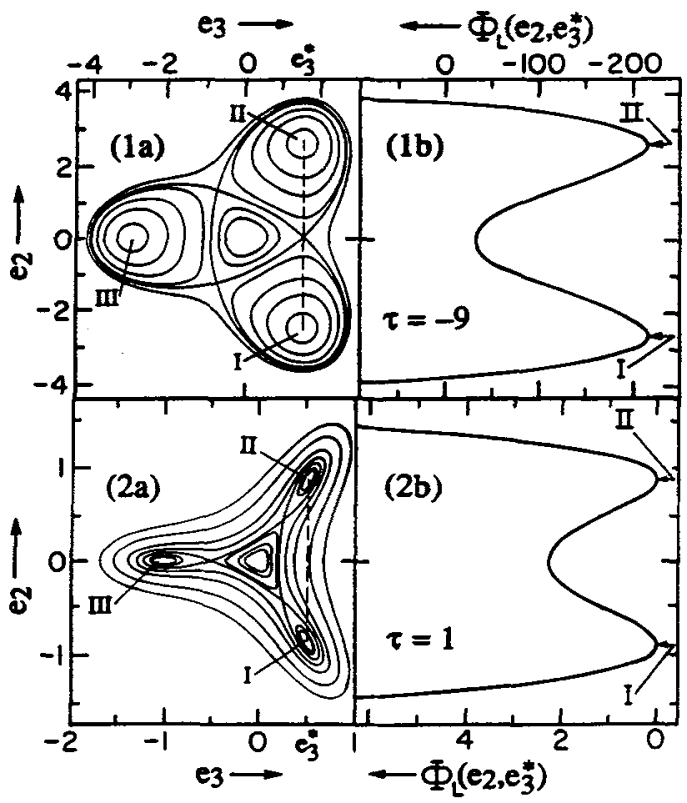

$$
\Phi_{\mathrm{L}}^{*}(\mathrm{e})=\Phi_{\mathrm{L}}\left(\mathrm{e}, \mathrm{e}_{3}^{*}\right)-\Phi_{\mathrm{L}}\left(0, \mathrm{e}_{3}^{*}\right)=\mathrm{A}^{*} \mathrm{e}^{2}+2 \mathrm{Ce}^{4}
$$

is the simple double well potential shown in Figs. $1 \mathrm{~b}$ and $2 \mathrm{~b}$, and

$$
A^{*}=-(27 / 32) A_{0} z^{2}<0
$$

a temperature dependent negative effective elastic constant. The variational derivative of (6) gives the static solution $[13,14]$

$$
e=e_{0} \tanh s / \sqrt{2} \xi_{0} ; e_{0}=\sqrt{-A^{*} / 2 C}=\sqrt{3} e^{*} ; \xi_{0}=\sqrt{g /\left(-A^{*}\right)}
$$

Figure $3 b$ shows the strain profile according to $(9 a)$, and Fig. 3a the displacement field $u_{r}=u(s)$ obtained from $(9 a)$ with $e=-(1 / \sqrt{2})(d u / d s)$ and $e_{1}=0, e_{3}=e_{3}^{*}$ by integration, resulting in the "V"-shape of the bicrystal.

Figure 3. (a) Shape of a bicrystal with a smooth twin boundary of width $2 \xi_{0}$. The tangential surface forces $T_{r}^{+}, T_{r}$ are required to sustain frictionless motion of the boundary, and/or to overcome internal friction, when moving it in the s-direction. (b) Strain profile at rest [static solution, Eq. (9)]. (c) Strain profile for frictionless motion, case (ii). (d) Strain profile for dissipation dominated motion, case (iii).

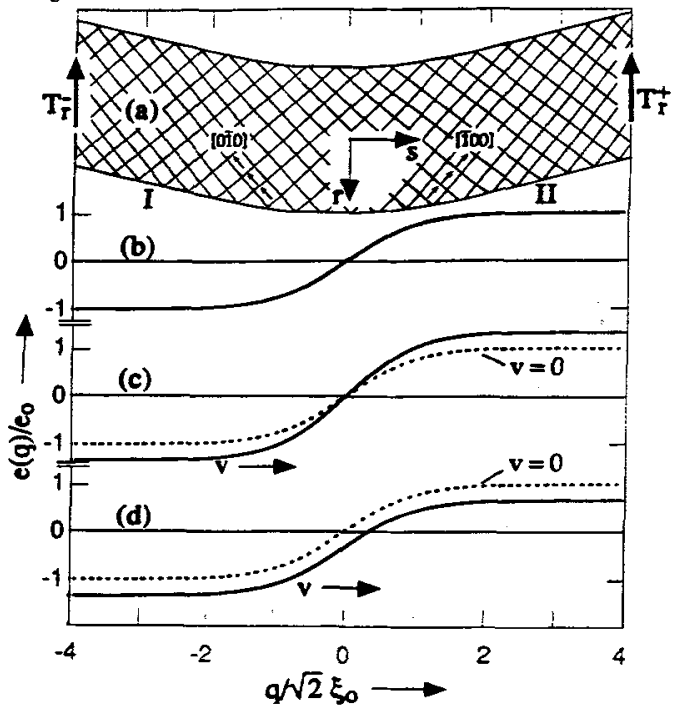




\subsection{Phonon Viscosity Model: Equation of Motion and Boundary Conditions}

The above assumptions (i) to (iv), used in [13] and [14] for the static solutions, are also sufficient to obtain an analytic solution for a moving TB. In addition, thermal effects will be ignored in the following, and the difference between isothermal and adiabatic elastic constants is neglected. In the coordinate system considered the motion of a TB (initially located at $s=0)$ in the $+s$ direction requires that for $s \leqslant-\xi(+\xi \leq s)$ the material particles move in the $-r(+r)$ direction, respectively (cf. Fig. 2a). Therefore, with $u_{r}=u(s, t)$ the kinetic energy density is given by $(\rho / 2)(\partial u / \partial t)^{2}$. On the basis of the phonon viscosity model the dissipation energy density is [19] $(\eta / 2)\left(\partial^{2} u / \partial s \partial t\right)^{2}$, where $\eta=\left(\eta_{11}-\eta_{12}\right) / 2$ is the phonon viscosity coefficient for a (time dependent) (110)/[110] shear deformation. Therefore, the equation of motion for the transverse displacement $u(s, t)$ that describes a moving TB is obtained from Eq. (6) [with $\left.e=-(1 / \sqrt{2}) u^{\prime}\right]$ in conjunction with the kinetic and the dissipative energy densities and becomes [19]

$$
\rho \ddot{u}-\eta \dot{u}^{\prime \prime}-A^{*} \mathbf{u}^{\prime \prime}-3 C\left(\mathbf{u}^{\prime}\right)^{2} \mathbf{u}^{\prime \prime}+g^{\prime \prime "}=0
$$

where $\left(^{\circ}\right)=(\partial / \partial \mathrm{t})$ and ()$^{\prime}=(\partial / \partial s)$. Considering a rectangular parallelepiped with edges of length $2 \mathrm{~L}_{\mathrm{T}}, 2 \mathrm{~L}_{\mathrm{S}}$ and $2 \mathrm{~L}_{3}$ that is centered at the origin of the $\left(r, \mathrm{~s}, \mathrm{x}_{3}\right)$-coordinate system, integrating Eq. (10) over $s$ from $-\mathrm{L}_{s}$ to $+\mathrm{L}_{\mathrm{s}}$ and assuming a steady state travelling wave solution of the form

$$
\mathrm{u}=\mathrm{u}(\mathrm{q}) ; \mathrm{q}=\mathbf{s}-\mathrm{vt}
$$

gives

$$
\rho v^{2} e+\eta v e^{\prime}-A^{*} e-3 C e^{3}+g e^{\prime \prime}=\chi
$$

Requiring that, as for the static solution (a), also for a moving TB the strain assumes constant values at large distances from the boundary implies that, with $\xi<<\_\mathrm{L}_{\mathrm{s}}$ for $\mathrm{q} \rightarrow \pm \ell$ it is $\mathrm{e}(\mathrm{q}) \rightarrow \mathrm{e}_{+}, \mathrm{e}_{-}$(=const), and $\mathrm{e}^{\prime} \rightarrow 0, \mathrm{e}^{\prime \prime} \rightarrow 0$. Then $\mathrm{e}_{+}, \mathrm{e}_{-}$are determined by the surface forces and hyperforces at $\mathrm{s}= \pm \mathrm{I}_{\mathrm{s}}$ (cf. Eqs. (2.22) to (2.24) of Ref. [20]). For the LG free energy (6) the only nonzero components are the tangential forces $T_{r}^{+}$and $T_{r}^{-}$determined by

$$
\mathrm{T}_{\mathrm{r}}^{+} / \sqrt{2}=-\left(\mathrm{A}^{*} \mathrm{e}_{+}+2 \mathrm{C} \mathrm{e}_{+}^{3}\right) ; \mathrm{T}_{\mathrm{r}}^{-} / \sqrt{2}=+\left(\mathrm{A}^{*} \mathrm{e}_{-}+2 \mathrm{Ce}_{-}^{3}\right)
$$

In addition, one obtains from Eq. (13) for $q \rightarrow \pm \infty$

$$
\rho v^{2}\left(e_{+}-e_{-}\right)=-\left(T_{r}^{+}+T_{r}^{-}\right) / \sqrt{2} ; \chi=\left(T_{r}^{+}-T_{r}^{-}\right) / 2 \sqrt{2}+\frac{\rho v^{2}}{2}\left(e_{+}+e_{-}\right)
$$

These four equations determine the four quantities $e_{+}, e_{-}, v$ and the integration constant $\chi$ in terms of the surface forces $T_{\mathbf{r}}^{+}$and $T_{\mathbf{r}}^{-}$.

\subsection{Travelling Soliton Solution}

Since the standard procedure for solving equations for the type represented by Eq. (12) [i.e. integration after multiplication with e'] does not work here [because of the presence of the dissipative term $\left.\sim \mathrm{e}^{\prime}\right]$ we use the trial function (see also Ref. [21])

$$
e(q)=\frac{1}{2}\left(e_{+}+e_{-}\right)+\frac{1}{2}\left(e_{+}-e_{-}\right) \tanh q / \sqrt{2} \xi
$$

which satisfies the boundary condition $\lim _{\ell \rightarrow \infty} e( \pm \ell)=e_{ \pm}$. By inserting (15) into (12) one verifies that (15) is indeed a solution of (12) if the following additional relations are satisfied:

$$
\begin{aligned}
& e_{+}^{2}+e_{-}^{2}+e_{+} e_{-}=e_{0}^{2}\left[1+\left(v / v_{0}\right)^{2}\right] \\
& \left(e_{+}+e_{-}\right) e_{+} e_{-}=x / 2 C \\
& v=-\left(\sqrt{3} / 2 e_{0}\right) v_{1}\left(e_{+}+e_{-}\right) \operatorname{sgn}\left(e_{+}-e_{-}\right) \\
& \xi=\xi_{0}\left[1+\left(1-\lambda^{2}\right)\left(v / v_{0}\right)^{2}\right]^{-1 / 2}
\end{aligned}
$$

Here 


$$
v_{0}=\sqrt{ }-A^{*} / \rho ; v_{1}=\sqrt{6 g\left(-A^{*}\right)} / \eta ; \lambda=\left(v_{d} / v_{1}\right)=\eta / \sqrt{ } 6 \rho g
$$

are material parameters representing two characteristic velocities and their ratio: $v_{0}$ is related to the velocity $v_{t}$ of a [110]/[110] transverse wave in the tetragonal phase $\left[v_{t}=\sqrt{2} v_{0}\right]$, and $v_{1}^{-1}$ is a measure of the viscosity appropriate for the present problem. The relations $(16 a, b)$ can be derived from $(13 a, b)$ and $(14 a, b)$ by eliminating $T_{\mathrm{r}}^{+}$and $\mathrm{T}_{\mathrm{r}}$. Their physical significance will be discussed elsewhere. Equation (17) is an additional relation and provides a constraint on $\mathrm{T}_{\mathrm{r}}^{+}$and $\mathrm{T}_{\mathrm{r}}^{-}$to the effect that $\left(\mathrm{T}_{\mathrm{r}}^{+}+\mathrm{T}_{\mathrm{r}}^{-}\right)$becomes a single valued function of $\left(T_{r}^{+}-T_{r}\right)$ that is in the small velocity limit $\left(v<<v_{0}\right)$ given by

$$
\left(\mathrm{T}_{\mathrm{r}}^{+}+\mathrm{T}_{\mathrm{r}}^{-}\right) / 2 \mathrm{C}=-\left(3 / 8 \sqrt{2} \mathrm{e}_{0}^{3} \lambda^{2}\right)\left[\left(\mathrm{T}_{\mathrm{r}}^{+}-\mathrm{T}_{\mathrm{r}}^{-}\right) / 2 \mathrm{C}\right]^{2} \operatorname{sgn}\left(\mathrm{e}_{+}-\mathrm{e}_{-}\right)
$$

Expressing the strains also in terms of their sum and difference one obtains

$$
\begin{aligned}
& \left(e_{+}+e_{-}\right)\left\{\left[1-\left(9 / 8 \lambda^{2}\right)\right]\left(e_{+}+e_{-}\right)^{2}-e_{o}^{2}\right\}=\left(T_{r}^{+}-T_{r}^{-}\right) / 4 \sqrt{2} C \\
& \left(e_{+}-e_{-}\right)= \pm 2 e_{o}\left[1+\left(1-\lambda^{2}\right)\left(v / v_{0}\right)^{2}\right]^{1 / 2}
\end{aligned}
$$

The four relations (17), (18) and (21a,b) completely determine the parameters that enter the solution (15).

\subsection{Special Cases}

(i) For $T_{T}^{+}=T_{r}^{L}=0$ the static solution $\left[v=0, e_{+}=-e_{-}=e_{o}\right]$ of Eqs. $(9 a, b, c)$ is obtained.

(ii) For $\eta=0$ (frictionless motion) one obtains $\mathrm{e}_{+}=-\mathrm{e}_{-}=\mathrm{E}(\mathrm{v})=\mathrm{e}_{\mathrm{o}}\left[1+\left(\mathrm{v} / \mathrm{v}_{\mathrm{o}}\right)^{2}\right]^{1 / 2}, \mathrm{~T}_{\mathrm{t}}^{+}=\mathrm{T}_{\mathrm{t}}^{-}=-\sqrt{2} \mathrm{pv^{2 }} \mathrm{E}(\mathrm{v})$, $\xi=\xi_{0}\left[1+\left(v / v_{0}\right)^{2}\right]^{-1 / 2}$ and $e(q)=E(v) \tanh (q / \sqrt{2} \xi)$ [cf. Fig. 3c]. The equality of the two surface forces signifies that there is no driving force $\left(\mathrm{T}_{\mathrm{r}}^{+}-\mathrm{T}_{\mathrm{r}}\right)$ and that the surface force is required only to balance the inertial (centrifugal) force that arises over the width $2 \xi$ of the TB as a result of the reversal of direction of the

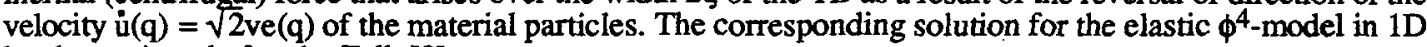
has been given before by Falk [8].

(iii) For $v<<v_{1}<(4 / 3) v_{0}$ (viscosity dominated low velocity limit) one obtains to order $O\left[\left(v / v_{0}\right)^{2}\right]$ with $T_{r}^{+}$ $=-\mathrm{T}_{\mathrm{r}}+\mathrm{O}\left[\left(\mathrm{v} / \mathrm{v}_{\mathrm{O}}\right)^{2}\right]$

$$
\begin{aligned}
& e(s)=\frac{1}{2}\left(e_{+}+e_{-}\right)+e_{o} \tanh q / \sqrt{2} \xi_{o} \\
& e_{+}+e_{-}=-\left(T_{r}^{+}-T_{r}^{-}\right) / 2 \sqrt{2}\left(-A^{*}\right) \\
& v=(3 / 4)\left(\xi_{o} / e_{o} \eta\right)\left(T_{r}^{+}-T_{r}^{-}\right) \\
& e_{ \pm}= \pm e_{o}\left\{1 \mp e_{o} \eta v /\left[3 \sqrt{2} \xi_{o}\left(-A^{*}\right)\right]\right\}
\end{aligned}
$$

Eqs. $(22 \mathrm{a}, \mathrm{b})$ show that in this case the strain curve for the static solution is simply shifted vertically by an amount proportional to the driving force [cf. Fig. 3d]. Equation (22c) shows that for $\mathrm{T}_{\mathrm{r}}^{+}>0, \mathrm{~T}_{\mathrm{r}}^{-}<0$ (i.e. $-T_{r}^{+}, T_{r}^{-}$are upward in Fig. 3 for the coordinate system used) the TB moves to the right $(v>0)$, and Eqs. $(22 \mathrm{~d}, \mathrm{e})$ show that in the homogeneous regions $\mathrm{q} \rightarrow+\infty(-\infty)$ the strain is reduced (increased) by an amount proportional to $\eta v$, respectively.

(iv) The general solution of Eqs. (17) and $(21 \mathrm{a}, \mathrm{b})$ depends on the parameter $\lambda$ and leads to a nonlinear dependence of the TB velocity on the driving force $\left(\mathrm{T}_{\mathrm{r}}^{+}-\mathrm{T}_{\mathrm{r}}^{-}\right.$); it will be discussed elsewhere and compared with similar results obtained by Abeyaratne and Knowles $[9,10]$ on the basis of a tri-linear stress-strain relation [instead of the smooth relations (13a,b)].

\section{APPLICATION TO VANADIUM SILICIDE}

The three material parameters that determine according to (22c) the intrinsic $\mathrm{TB}_{\mathrm{B}}$ mobility $\mu=v /\left(\mathrm{T}_{\mathrm{r}}^{+}-\mathrm{T}_{\mathrm{r}}\right)$ may be determined from experimental crystallographic, elastic, anelastic and phonon dispersion data $[13,14,22]$. $\mathrm{V}_{3} \mathrm{Si}$, which undergoes a proper (or "pseudo-proper") FE $\mathrm{O}_{\mathrm{h}}-\mathrm{D}_{4 \mathrm{~h}}$ transformation at about $21 \mathrm{~K}$ [23] is the only such material for which, at least in principle, all necessary experimental input data are available. Using the same procedures as before for other proper FE martensites [13,22], one calculates from Refs. [23] and [24] a transformation strain of $e_{0}=9.4 \times 10^{-5}$, and from Refs. [24] and [25] for the TB half-width $\xi_{0}=$ $0.36 \mathrm{~nm}$, both referred to the transformation temperature. The TB width is only four times larger than the shortest interplanar distance in [110] and should decrease with decreasing temperature [13], suggesting the existence of a mobility threshold due to the Peierls barrier for $\mathrm{T}<\mathrm{T}_{0}$. 
Calculating $\eta$ from the measured ultrasonic attenuation $\alpha$ [24] by means of the relation for the phonon viscosity model, $\alpha=\eta \omega^{2} /\left(2 \rho v_{s}^{3}\right.$ ) (where $\omega=$ angular frequency and $v_{s}=$ sound velocity for a [110]/[110] shear wave) gives for $\eta$ (in $\mathrm{Nsec} / \mathrm{m}^{2}$ ) 0.015 at $300 \mathrm{~K}$, a maximum of 0.047 at $50 \mathrm{~K}$, and 0.032 at $25 \mathrm{~K}$, corresponding to a mobility value of about $10^{-4} \mathrm{~m}^{3} / \mathrm{Nsec}$ at $\mathrm{T}_{0}$. However, the values given for $\eta$ are $10^{3}$ to $10^{4}$ times larger than theoretically calculated room temperature values for seven metals [15], so that the ultrasonic attenuation measured for $\mathrm{V}_{3} \mathrm{Si}$ is certainly not directly due to phonon viscosity. For $\mathrm{Cu}, \mathrm{Al}$ and $\mathrm{Pb}$ experimental ultrasonic attenuation data have been accounted for in terms of separate contributions from the dislocation drag that is caused by phonon viscosity, electronic damping (dominant only at low temperatures) phonon scattering, and (for longitudinal modes) from the thermoelastic effect [26]. Interpretation of the attenuation data for $\mathrm{V}_{3} \mathrm{Si}$ [24] in this manner would require measurement of the frequency dependence of the attenuation as a function of temperature. Additional contributions from pretransformation strain modulations (tweed) observed in $\mathrm{V}_{3} \mathrm{Si}$ [27] (as in many other thermoelastic martensites) above the transformation temperature are also to be expected.

\section{Acknowledgments}

The author expresses his gratitude to G. B. Olson (Northwestern University, Evanston) for bringing the problem of the mobility of martensitic interfaces to his attention, and to J. K. Knowles (California Institute of Technology, Pasadena) and L. Truskinovsky (University of Minnesota, Minneapolis) for sending preprints of their work prior to publication.

\section{References}

[1] Nishiyama, Z., Martensitic Transformation (Academic Press, New York, 1978) pp. 232-238.

[2] Grujicic, M., Olson, G. B. and Owen, W. S., Metall. Trans. 16A (1985) 1713-1722.

[3] Otsuka, K., Wayman, C. M., Nakai, K., Sakamoto, H. and Shimizu, K., Acta Met. 24 (1976) 207-

226.

[4] Grujicic, M., Olson, G. B. and Owen, W. S., Metall. Trans. 16A (1985) 1723-1734.

[5] Olson, G. B. and Cohen, M., Acta Met. 27 (1979) 1907-1918; ibid. 29 (1981) 1475-1484.

[6] Kocks, U. F., Argon, A. S. and Ashby, M. F., Progress Mat.Sci. 19 (1975) 1-288.

[7] Grujicic, M., Olson, G. B.and Owen, W. S., Metall. Trans. 16A (1985) 1735-1744.

[8] Falk, F., J. Phys. C: Solid State Phys. 20 (1987) 2501-2509.

[9] Abeyaratne, R. and Knowles, J. K., SIAM J. Appl. Math. 51 (1991) 1205-1221.

[10] Abeyaratne, R. and Knowles, J. K., "Nucleation, kinetics and admissibility criteria for propagating phase boundaries," Shock Induced Transitions and Phase Structures in General Media, Minneapolis, MN, Oct. 1990, J. E. Dunn, R. Fosdick, and M. Slemrod, Eds. (Springer, New York, 1993) pp. 1-33.

[11] Truskinovsky, L., "Kinks versus shocks," see Ref. [10], pp. 185-229.

[12] Barsch, G. R. and Krumhansl, J. A., Phys. Rev. Lett. 53 (1984) 1069-1072.

[13] Barsch, G. R. and Krumhansl, J. A., "Topological Soliton Models for Interfaces in Proper Ferroelastic Martensites," Martensitic Transformations (ICOMAT-92), Monterey, CA, 20-24 July 1992, C. M. Wayman and J. Perkins, Eds., (Monterey Inst. Adv. Studies, Monterey, CA, 1993), pp. 53-64.

[14] Barsch, G. R., Cao, W. C. and Krumhansl, J. A., Phys. Rev. B53 (1996) to be published.

[15] Mason, W. P., J. Acoust. Soc. America 32 (1960) 458-472.

[16] Horovitz, B., Barsch, G. R.and Krumhansl, J. A., Phys. Rev. B43 (1991) 1021-1033.

[17] Mason, W. P., Physical Acoustics and the Properties of Solids (Van Nostrand, Princeton, 1958) pp.

187-193.

[18] Woodruff, T. O. and Ehrenreich, H., Phys. Rev. 123 (1961) 1553-1559.

[19] Landau, L. D. and Lifshitz, E. M., Theory of Elasticity (Pergamon, Oxford, 1970), pp. 153-155.

[20] Barsch, G. R. and Krumhansi, J. A., Metall. Trans. 19A (1988) 761-775.

[21] Collins, M. A., Blumen, A., Currie, J. F. and Ross, J., Phys. Rev. B19 (1979) 3630-3644.

[22] Donovan, D. W., Barsch, G. R., Pangborn, R. N. and Finlayson, T. R., Acta Metall. Mater. 42 (1994) 1985-1996.

[23] Batterman, B. W. and Barrett, C. S., Phys. Rev. 145 (1966) 296-301.

[24] Testardi, L. R. and Bateman, T. B., Phys. Rev. 154 (1967) 402-410.

[25] Shirane, G., Axe, J. D. and Birgeneau, R. J., Solid State Comm. 9 (1971) 397-400.

[26] Mason, W. P., "Low- and High-Amplitude Internal-Friction Measurements in Solids and their Relation to Imperfection Motions," in: Microplasticity, C. J. McMahon, Jr., Ed. (Interscience/Wiley, New York) pp. 287-364.

[27] Onozuka, N., Ohnishi, N. and Hirabayashi, M., Metall. Trans. 19A (1988) 797-801. 